\title{
A dislocation dynamics study of the strength of stacking fault tetrahedra. Part II: interactions with mixed and edge dislocations
}

\author{
E. Martinez , J. Marian and J.M. Perlado \\ Lawrence Livermore National Laboratory, 7000 East Avenue, Livermore, CA \\ Instituto de Fusión Nuclear, Universidad Politécnica de Madrid, Spain
}

\begin{abstract}
In this paper we present the sequel to Part I and present a comprehensive dislocation dynamics study of the strength of stacking fault tetrahedra to mixed and edge dislocation glides in $\mathrm{fcc} \mathrm{Cu}$.
\end{abstract}

Keywords: dislocation dynamies; irradiation damage; stacking-fault tetrahedron; mechanical properties; $\mathrm{Cu}$

\section{Introduction}

This paper is the second part of our dislocation dynamics (DD) study on screw dislocation interactions with stacking-fault tetrahedra (SFTs) in $\mathrm{Cu}$ (hereafter referred to as Part I). Here we study edge and $60^{\circ}$ and $30^{\circ}$ mixed dislocations interacting with SFTs ranging from 2.5 to $4.7 \mathrm{~nm}$ in size. As in Part I, the potential parametric space to be explored is multi-dimensional, including SFT size and orientation, dislocation line length and character, Burgers vector direction, number of non-zero stress tensor components, intersection between the dislocation glide plane and the tetrahedron, etc. Here we use DD simulations to study dislocation-SFT strength as a function of the distance between the dislocation glide plane and the tetrahedron's base, dislocation line length, and Burgers vector orientation, and, together with Part I, this work completes the study as a function of dislocation character. In each case, we identify the corresponding mechanisms in terms of partial dislocation reactions and relate our findings to the current understanding in irradiated fcc microstructures. Our objective is that the combined conclusions extracted from this and our previous study on screw dislocations will be helpful in interpreting fine-scale experimental observations or other more 'macroscopic' measurements such as yield stress increases and/or ductility loss in irradiated fcc metals.

There have been several recent molecular dynamics (MD) simulations of edge dislocation-SFT interactions in $\mathrm{Cu}$. For example, Osetsky et al. have reported that the critical resolved shear stress (CRSS) required to traverse an SFT increases as the glide plane of an edge dislocation gets closer to the base of the SFT and the temperature decreases Also, Wirth et al. have shown that SFTs are neither absorbed nor destroyed, but sheared, by an edge dislocation They find that successive dislocation passages can result in further shearing of the tetrahedron, leading to separation into two 
pieces about the dislocation glide plane. Lee et al. have recently performed MD simulations where edge dislocations carry out an elaborate reaction-driven interaction with 4.7-nm SFTs, leading to structures similar to those left by screw dislocations [7]. In contrast, the amount of available atomistic simulations on mixed dislocations is quite limited $[7,8]$. This is primarily due to the difficulties associated with non-orthorhombic boundary conditions, which complicates the simulations' set up in terms of computational box orientation, stress (or strain) application, etc. In any case, time and length scale limitations, and associated artifacts - strain-rate effects, image forces, etc. - limit the amount of statistically significant information that can be extracted from MD studies.

These difficulties are virtually non-existent in DD and the study of dislocation-SFT interactions as a function of dislocation character is trivial from a dislocation dynamics point of view. Two aspects need to be kept in mind to ensure the fidelity of DD simulations for such a study, however, namely, that the assumption of linear elasticity hold, and that partial dislocations be explicitly considered. We have solved both of these issues in our previous work and, to avoid redundancies, in his paper we omit the methodology section and move directly onto the results section, followed by a discussion on the validity of our results.

\section{Results}

Following the outline of Part I, below we present the results of the interaction of mixed and edge dislocations with a SFT as given by DD calculations. We calculate the strength of SFTs as a function of dislocation character, length, reacting geometry, and SFT size. We start by providing a detailed description of the geometry and boundary conditions employed.

\subsection{Interaction geometry and boundary conditions}

The geometry of the Thompson tetrahedron dictates the nature of the possible interaction configurations. As we have shown in Part I, screw dislocations lend themselves to 'perfect' face and edge-on interactions. The same is true for $60^{\circ}$ mixed dislocations, but for edge and $30^{\circ}$ mixed dislocations there is only one possible interaction geometry (shown in Figure 1). Moreover, as we shall see, all interactions can in principle be reduced to a few basic partial dislocation reactions stemming from pure screw $/ 60^{\circ}$-mixed dislocations face and edge-on. Our simulations consist of (initially) straight dislocation segments of length $L$ with fixed end points, i.e. akin to having a Frank-Read source operating on a single slip system. This is different to most MD simulations, which use periodic boundary conditions. The initial geometry is shown in Figure 2a.

In the case of $60^{\circ}$-mixed dislocations, the face-on orientation brings partial and perfect dislocations directly in contact with one of the facets of the tetrahedron. How this contact evolves under applied pure shear stress depends on the resistance exerted by such facets on Shockley partials with a net force component in the direction of the facet plane normal. This resistance is characterized by the minimum stress, $\sigma_{\mathrm{SF}}$, required by a dislocation partial to traverse a stacking fault surface. This stress will determine the threshold after which a dislocation can penetrate the tetrahedron, shearing it. In Part I we have computed a value of $\sigma_{\mathrm{SF}} \approx 1659 \mathrm{MPa}$ for screw dislocations and here we perform a similar calculation for $60^{\circ}$ dislocations. Unlike screws, which dissociate into two Shockley partials 



Figure 1. Geometry of a perfect edge and $30^{\circ}$ mixed dislocation (looking down onto the glide plane $(d)$ ) with respect to an SFT. In both cases, this geometry only allows for a single possible orientation. (1) Schematic diagram of the initial configuration. $h_{0}$ and $h$ are, respectively, the SFT size and the glide plane height (seen here in perspective with respect to the SFT base). (2) Configuration after the dislocation collides and 'hugs' the tetrahedron. Both types of dislocations become a perfect screw on one facet and a $60^{\circ}$ mixed dislocation on the other two. Note that this is an illustrative cartoon and the depicted line shapes do not correspond necessarily to the equilibrium ones.

that create equivalent stress fields, $60^{\circ}$-mixed dislocations dissociate into Shockleys with Burgers vectors that have different edge and screw components. Here we have studied both orientations and taken the most conservative one for our purposes (the one that leads to a lower $\left.\sigma_{\mathrm{SF}}\right)$. Calculations are performed by minimizing the energy of atomistic structures containing a (dissociated) $60^{\circ}$-mixed dislocation dipole and a stacking fault surface. Shear stress is applied only on the glide plane using a Parrinello-Rahman algorithm [10]. For such a configuration, we obtain $\sigma_{\mathrm{SF}}=1240.5 \mathrm{MPa}$ for the most conservative dissociation, $\approx 24 \%$ lower than the value obtained for screw dislocations. This corresponds to a nodal force per lattice parameter of $f^{*}=8.96 \times 10^{-11} \mathrm{~N}$. This force is then implemented as a Heaviside step function located at the SFT's facets.

Prior to studying the strength of SFTs to edge and mixed dislocation glide, we perform a quick check to test whether local constriction is achieved for a 70 -nm $60^{\circ}$-mixed dislocation before $\sigma_{\mathrm{SF}}$ is overcome. Results are shown in Figures 2(b) and (c), with full constriction being attained before $f^{*}$ is surpassed. We have found this behaviour to hold for all interaction heights $h$.

\subsection{Interaction mechanism between $60^{\circ}$ mixed dislocations and SFTs}

The interaction mechanism of a $60^{\circ}$ mixed dislocation impinging face-on with an SFT bears significant similitude with that for screw dislocations described in Part I. The main difference is that the initial dissociation, shown in frame (1) of Figure 3, results in a Shockley partial plus a Frank partial, rather than a simple re-dissociation into Shockleys on the plane of the SFT facet. All other aspects of the interaction mechanism remain 
(a)

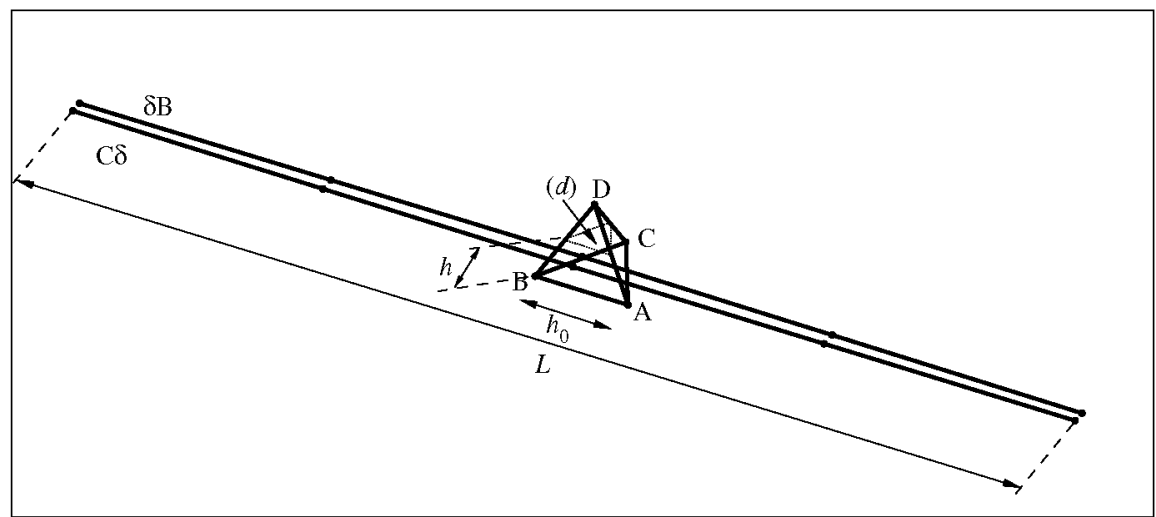

(b)

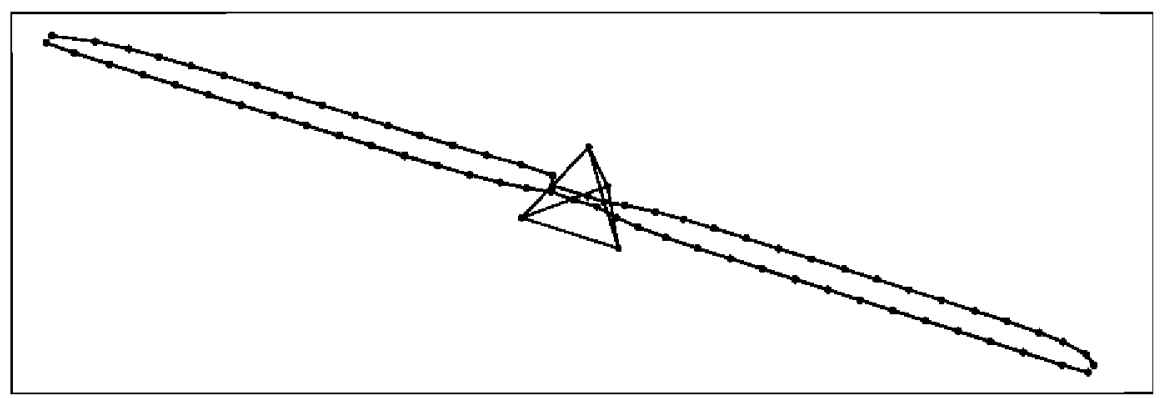

(c)

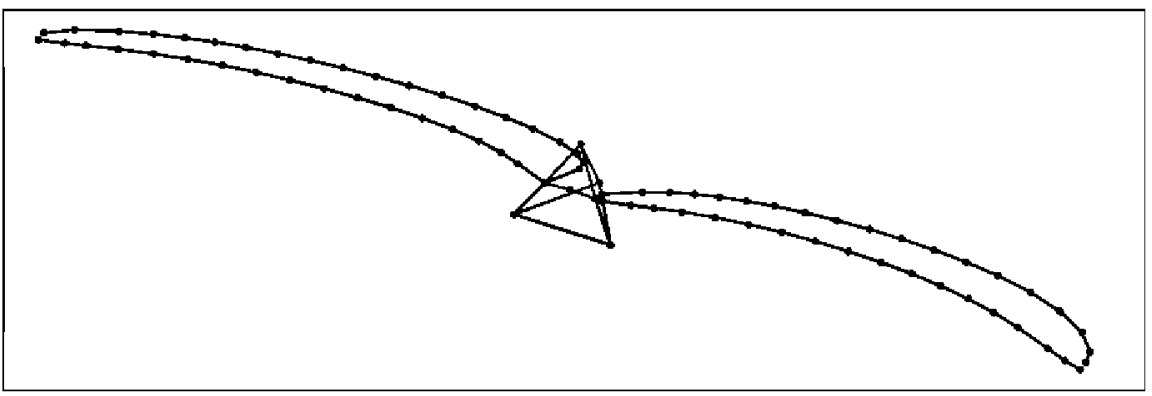

Figure 2. Sequence of snapshots from a DD simulation of a dissociated $60^{\circ}$-mixed dislocation being driven towards an SFT at its mid-height plane face-on. (a) Initial geometry including the tetrahedron orientation, the dislocation splitting and the area of the SFT intersected by the glide plane (labelled ' $\left.(d)^{\prime}\right) . L$ is the separation between the pinning points of the Frank-Read source, while $h$ and $h_{0}$ are defined as in Figure 1. (b) The leading partial is arrested upon contact with the SFT facet, prompting collapse from the trailing partial. (c) Full constriction is attained before $f^{*}$ is surpassed. Dislocation segments are coloured according to their Burgers vector: Shockley partials in blue, stair-rods in green, and perfect dislocations in red. For colour, see online.

fundamentally the same. In fact the surviving structure after dislocation passage, shown in Figure 3(6), is of the same type as that shown in frame (6) of Figure 5 of Part I. The same can be essentially said of the edge-on interaction.

In the following we analyse the interaction mechanism in detail. We start with a $60^{\circ}$ mixed $\mathrm{CB}(d)$ dislocation, 70-nm long, gliding towards a $4.7 \mathrm{~nm}$ SFT. The applied stress is resolved only on the glide plane and, as for the screw dislocation case, the measured 

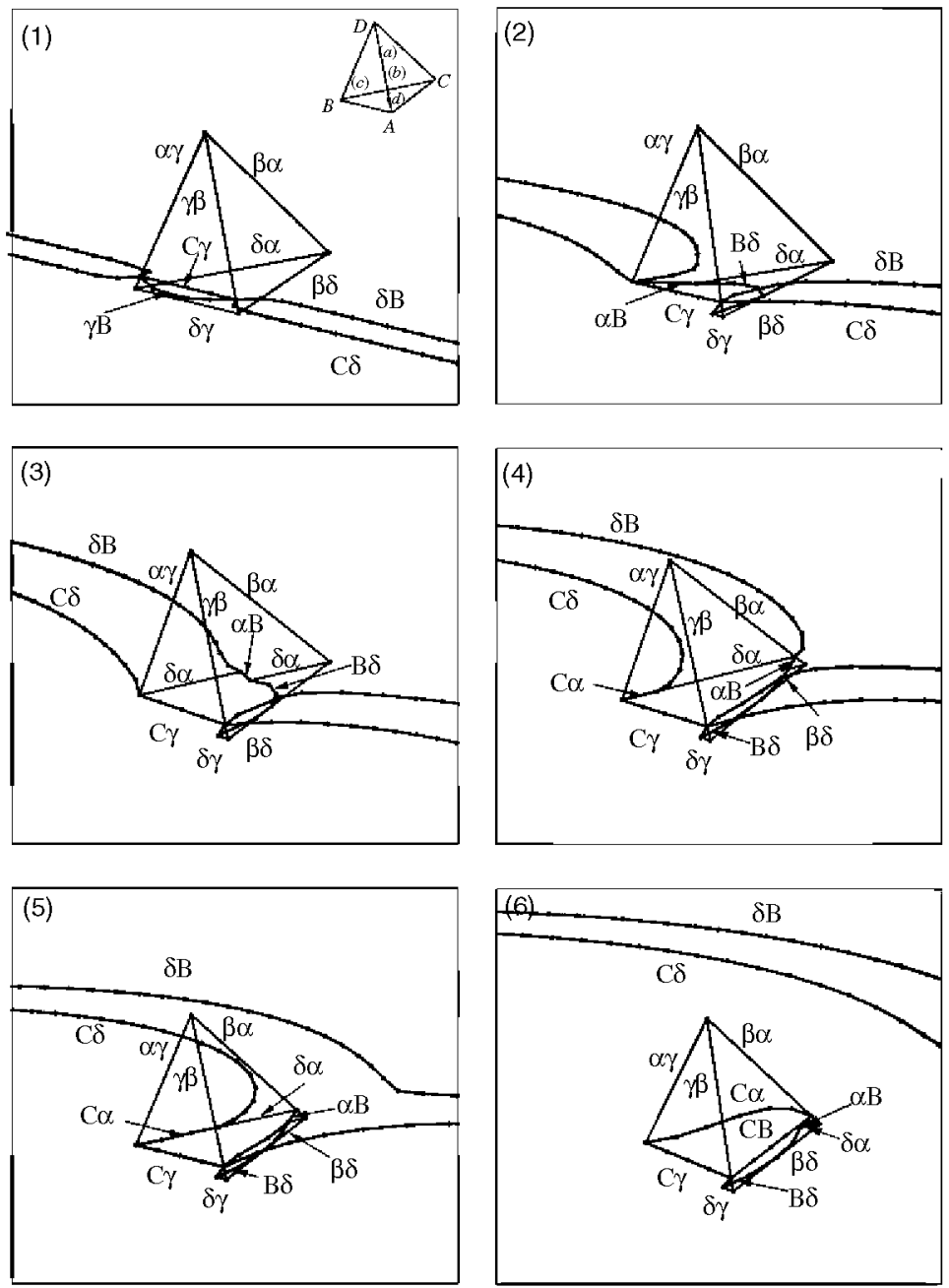

Figure 3. Sequence of simulation snapshots of the face-on interaction of a $60^{\circ}$ dislocation with a 4.7-nm SFT. See Section 2.2.1 for details.

dislocation velocities lie in the range $0.1-10 \mathrm{~m} \mathrm{~s}^{-1}$. We use Thompson's notation to describe all dislocation reactions.

\subsubsection{Face-on interaction mechanism}

As in Part I, shear stress is always applied incrementally so as to drive the dislocation into the SFT. Initial constriction on facet $(c)$ is always attained. The subsequent processes are described below, in direct correspondence with Figure 3:

(1) The perfect CB segment on facet (c) dissociates into a Frank dislocation, $\mathrm{C} \gamma$ and a Shockley partial $\gamma \mathrm{B}$ :

$$
\mathrm{CB} \rightarrow \gamma \mathrm{B}+\mathrm{C} \gamma
$$


(2) $\gamma \mathrm{B}$ glides down on the (c) plane and reacts with the basal stair-rod $\delta \gamma$, resulting in a new Shockley partial $\delta \mathrm{B}$ :

$$
\gamma \mathrm{B}+\delta \gamma \rightarrow \delta \mathrm{B}
$$

which results in the removal of the stacking fault (c) from the intersection height down. $\gamma \mathrm{B}$ reacts as well with the $\alpha \gamma$ stair-rod situated at the left-hand edge of $(c)$ :

$$
\gamma \mathrm{B}+\alpha \gamma \rightarrow \alpha \mathrm{B}
$$

(3) $\delta \mathrm{B}$ and $\alpha \mathrm{B}$ are glissile on facets $(d)$ and $(a)$, respectively, which they proceed to sweep, removing stacking fault in their wake. The outer segment of the leading partial then reacts with the new segment $\mathrm{B} \alpha$ to reform the stair-rod dislocation $\delta \alpha$ :

$$
\delta \mathrm{B}+\mathrm{B} \alpha \rightarrow \delta \alpha
$$

(4) The trailing partial catches up and its left-hand arm reacts with the newly-reformed $\delta \alpha$ stair-rod, giving rise to a $\mathrm{C} \alpha$ Shockley partial segment.

$$
\mathrm{C} \delta+\delta \alpha \rightarrow \mathrm{C} \alpha
$$

(5) The leading partial reconnects at the trailing edge of the SFT, laying down a segment of itself on $(b)$.

(6) The trailing partial then reconnects as well, leaving a constricted $\mathrm{CB}$ segment on (b). It is worth mentioning that the perfect and Frank segments, $\mathrm{CB}$ and $\mathrm{C} \gamma$, may easily dissociate changing this final structure. For example $\mathrm{C} \gamma$ could split into a $\delta \gamma$ stair-rod and a $\mathrm{C} \delta$ Shockley, which in turn could glide on $(d)$ (the original glide plane) and react with the $\mathrm{C} \alpha$ Shockley to form the missing stair-rod $\delta \alpha$. In this fashion, a smaller SFT could be formed, accompanied by a side structure.

When the applied stress is resolved only on the glide plane, the mechanism described above is valid for $h<0.15 h_{0}$. If the glide plane is above this value, the attraction between the basal stair-rod $\delta \gamma$ and the dissociated Shockley $\gamma \mathrm{B}$ is not sufficient to sustain the reactions in step (1) above. In this case, either an Orowan loop is left behind, or if $0.15 h_{0}<h<0.23 h_{0}$, a process similar to that of screw dislocations may occur on facet $(a)$.

When the Burger's vector of the initial mixed dislocation is $\mathrm{BC}$, the perfect segment formed on the $(c)$ facet of the SFT remains constricted due to the elastic repulsion between the basal stair-rod $\delta \gamma$ and the potential $\gamma$ B Shockley partial resulting from step (1) above. However, in such cases, the perfect screw segment created on facet $(a)$ when $h>0.15 h_{0}$ is amenable to dissociation, leading to a screw-type interaction mechanism.

\subsubsection{Edge-on interaction mechanism}

We now drive a $60^{\circ} \mathrm{CB}$ dislocation into a tetrahedron edge-on, such as depicted in Figure 4. The reaction starts with the leading Shockley partial C 8 'hugging' the SFT on facets $(b)$ and $(a)$. This leads to pinning and eventual constriction when the trailing partial reaches the tetrahedron. From there, the mechanism proceeds as follows:

(1) On facet (a), where the dislocation has perfect screw character, the following reaction occurs:

$$
\mathrm{CB} \rightarrow \alpha \mathrm{B}+\mathrm{C} \alpha
$$



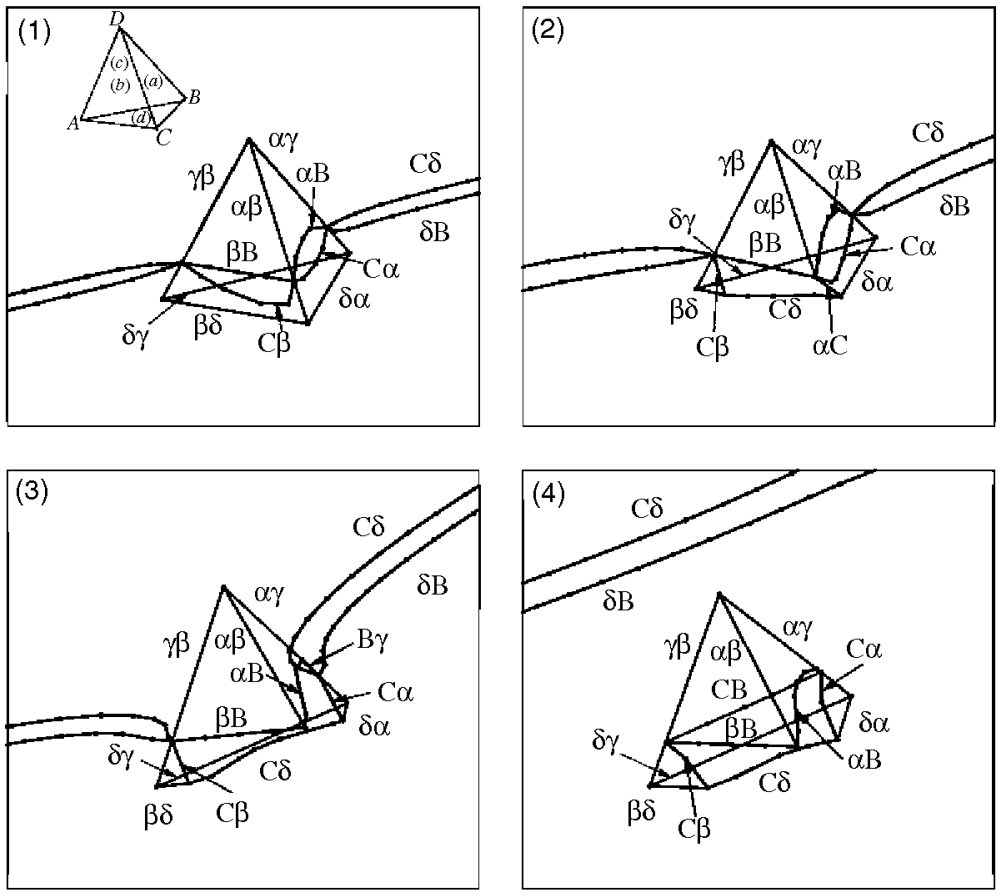

Figure 4. Sequence of simulation snapshots of the edge-on interaction of a $60^{\circ}$ dislocation with a 4.7-nm SFT. See Section 2.2.2 for details.

Conversely, on $(b)$ the dislocation is a $60^{\circ}$ mixed, so that the corresponding dissociation is:

$$
\mathrm{CB} \rightarrow \beta \mathrm{B}+\mathrm{C} \beta
$$

From there, the mechanism evolves as a combination of a screw and a $60^{\circ}$ mixed face-on (described, respectively in Part I and Section 2.2.1).

(2) The two Shockleys on $(b)$ and $(a)$ glide down their facets removing the stacking fault. When $\mathrm{C} \beta$ reaches the base, it reacts with the $\beta \delta$ stair-rod:

$$
\mathrm{C} \beta+\beta \delta \rightarrow \mathrm{C} \delta
$$

When it comes into contact laterally with the leading edge of the SFT, C $\beta$ has reversed its sign:

$$
\beta \mathrm{C}+\alpha \beta \rightarrow \alpha \mathrm{C}
$$

(3) The two Shockleys $\mathrm{C} \alpha$ and $\alpha \mathrm{C}$ annihilate one another while $\mathrm{C} \delta$ segment sweeps the SFT base.

(4) A minor increase in the level of stress makes the leading and trailing partials reconnect downstream of the SFT leaving a perfect dislocation segment in the back facet $(c)$. The final structure is similar to the one described above in Section 2.2.1. Again, the Frank segment may dissociate resulting in a reconstructed smaller SFT and a dissociated Frank loop by its side. 
This is the observed mechanism up to $h \leq 0.23 h_{0}$; above this height Orowan loops are produced. Inverting the Burgers vector from $\mathrm{CB}$ to $\mathrm{BC}$ modifies the mechanism slightly. The $60^{\circ}$-type dissociation (Frank + Shockley partial segments) on plane $(b)$ mentioned in step (1) does not take place. The dislocation remains constricted on that facet for the remainder of the interaction.

\subsubsection{Basal interaction mechanisms}

There are four possible interactions, the details of which we provide in Appendix A. We use the same geometries as above, i.e. $\mathrm{CB} / \mathrm{BC}(d)$ dislocations impinging on a 4.7-nm SFT. For clarity, here we summarize the most important results:

- Reaction CB face-on (Appendix A.1): the dislocation curves around the base, leaving an Orowan loop.

- Reaction BC face-on (Appendix A.2): the SFT is left intact.

- Reaction CB edge-on (Appendix A.3): the base is removed and the SFT is partially absorbed.

- Reaction BC edge-on (Appendix A.4): the base is removed and a perfect BC loop is left behind.

\subsubsection{Strength of SFTs to $60^{\circ}$-mixed dislocation passage}

The strength curves for the 4.7-nm SFT are given in Figure 5. Two important observations can be extracted from the curves: (i) edge-on interactions are harder than face-on interactions, and (ii) BC interactions show a stronger dependence with $h$ than those of CB. As for screw dislocations (Part I), dislocation line length appears to have a strong influence on the computed strengths. The inset to Figure 5 shows the strengths for $70-\mathrm{nm}$ segments compared to dislocation lines of $150 \mathrm{~nm}$. The data shown in the inset show clearly that the shape of the strength curves is not affected by the total dislocation line length, in fact it is simply rigidly translated. This suggests that the stress needed to overcome an SFT can be

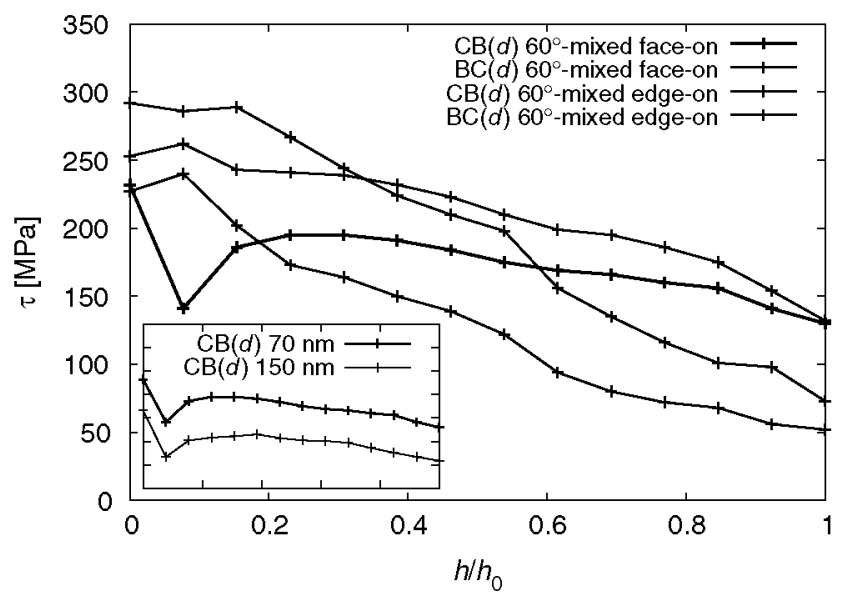

Figure 5. SFT strength as a function of the normalized interaction height, $h / h_{0}$, for a $60^{\circ}$-mixed dislocation impinging on a $h_{0}=4.7-\mathrm{nm}$ SFT face and edge-on. 
calculated using simple line tension arguments. Indeed, the ratio $\sigma^{\prime} / \sigma=0.54$ is reasonably close to the line length ratio of $L / L^{\prime}=70 / 150=0.47$ (the prime denotes results for $150-\mathrm{nm}$ dislocations). In other words, the interaction mechanisms do not affect the overall strengths, which are dictated by the dislocation line tension. This equivalence is expected to break down for dislocation lengths of the order of the tetrahedron size.

\subsubsection{SFT size effects}

Next we turn to the study of SFT strength to $70-\mathrm{nm}$ dislocations as a function of the tetrahedron size. Here, instead of plotting the results as in Figure 5 (as was the case in Figure 12 of Part I [1]), we express the data directly in terms of the areal stress function $\theta_{m}=\tau_{m} / a$ (where $a$ is the 'encountered' triangular area resulting from the intersection between the tetrahedron and the glide plane). In addition to the 4.7-nm data, results for 2.5 and 3.6-nm tetrahedra are shown in Figure 6. Again, all the data follow a clear trend in a logarithmic scale. This trend is best represented by a power law of the type $\theta_{m}(a)=B_{m} / a^{n_{m}}$ where $B_{m}$ and $n_{m}$ are constants and $a=\frac{\sqrt{3}}{4} l^{2} . l=h_{0}-h$ is the length of the edge of the traversed triangle. Upon fitting the $\theta_{m}$ function to the calculated data by means of a leastsquares fitting we obtain $B_{m}=160.82$ and $n_{m}=0.94$, very close to a $a^{-1}$ behaviour. From here, we derive a general expression for the shear stress that a $70-\mathrm{nm} 60^{\circ}$-mixed dislocation needs to traverse an SFT interacting face-on:

$$
\begin{gathered}
\theta_{m}(a)=\tau_{m}(a) / a=160.82 a^{-0.94} \\
\tau_{m}(a)=160.82 a^{0.06}
\end{gathered}
$$

The final form of $\tau_{m}(a)$ displays a weak dependence with $a$. The small error between the calculated data and the power law fit proves that the strength of an SFT is completely independent of its size - at least in the range of sizes explored - and that equal areas encountered in different size tetrahedra give rise to the same mechanical response.

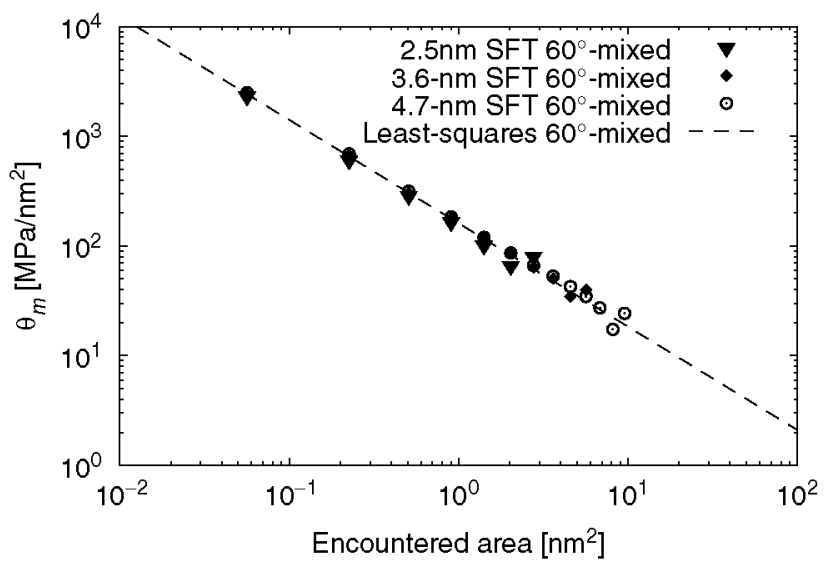

Figure 6. Variation of the stress per unit triangular area, $\theta_{m}$, with the area, $a$, encountered by a $60^{\circ}$ mixed dislocation. The curves show that the SFT strength is independent of the tetrahedron size, and depends solely on the area intersected by the dislocation glide plane. The least-squares fit to the data is $\theta_{m}(a)=160.82 / a^{0.94}$. 

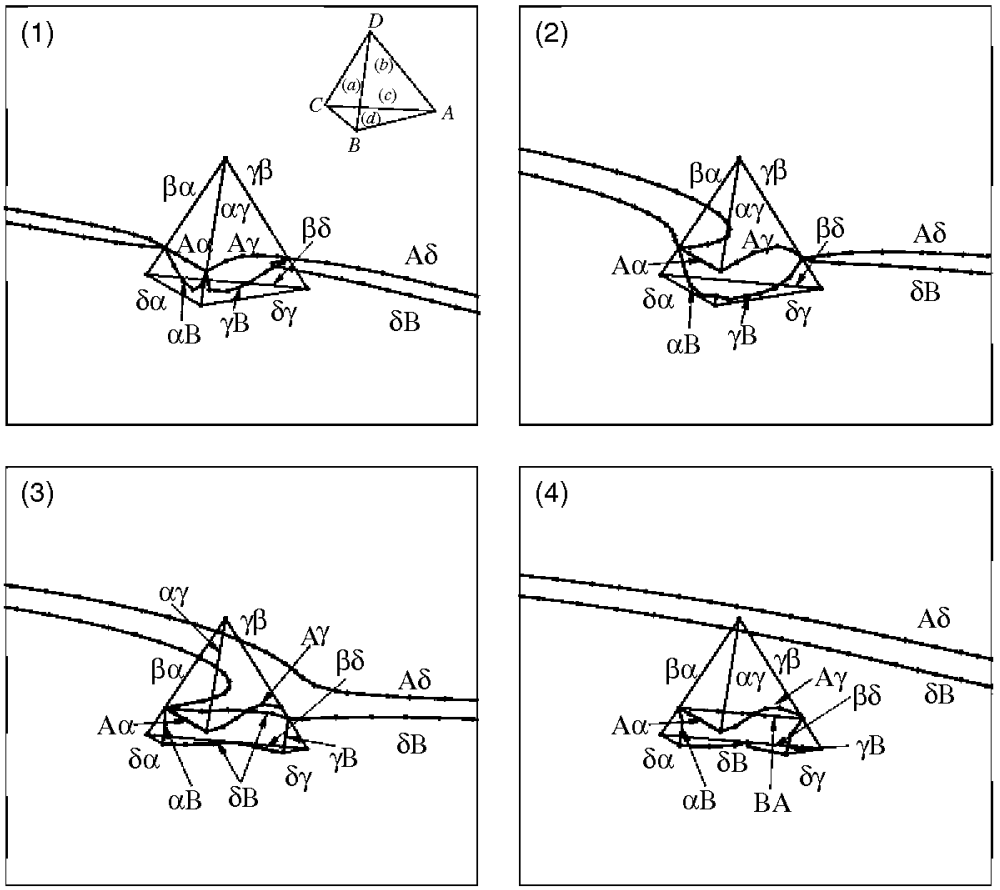

Figure 7. Interaction between an $\mathrm{AB}(d)$ edge dislocation and $a$ 4.7-nm SFT.

\subsection{Interaction mechanism between $30^{\circ}$ and edge dislocations and SFT's}

As Figure 1(1) shows, neither $30^{\circ}$ nor edge dislocations are aligned with any of the SFT facets or edges. Furthermore, both dislocations become pure screw on one facet and $60^{\circ}$ mixed on the other two. These geometric peculiarities simplify the treatment substantially, for we can reduce the entire analysis to studying only one of them. Here we choose edge dislocations interacting with an SFT according to the only configuration possible (shown in Figure 1).

\subsubsection{Non-basal interaction mechanism}

We start out with a perfect $\mathrm{AB}(d)$ edge dislocation being driven towards the SFT. The dislocation readily undergoes constriction on facets $(c)$ and $(a)$, becoming a perfect screw segment and a perfect $60^{\circ}$ mixed dislocation, respectively. These segments then dissociate following the reactions described respectively in Part I and Section 2.2.1. From there, the mechanism, shown in Figure 7, continues as follows:

(1) The dissociation of the perfect screw segment on $(c)$ is:

$$
\mathrm{AB} \rightarrow \gamma \mathrm{B}+\mathrm{A} \gamma
$$

On $(a)$, the $60^{\circ}$-mixed segment dissociates as:

$$
\mathrm{AB} \rightarrow \mathrm{A} \alpha+\alpha \mathrm{B}
$$


(2) The two Shockley partials gliding down on $(c)(\gamma \mathrm{B})$ and $(a)(\alpha \mathrm{A})$ meet at the edge dividing both facets (stair-rod $\alpha \gamma$ ), annihilating with it:

$$
\gamma \mathrm{B}+\mathrm{B} \alpha+\alpha \gamma \rightarrow 0
$$

(3) When $\gamma \mathrm{B}$ and $\alpha \mathrm{A}$ reach the base they react with the corresponding stair-rods, producing a $\delta \mathrm{B}$ Shockley that sweeps the base:

$$
\begin{aligned}
& \gamma \mathrm{B}+\delta \gamma \rightarrow \delta \mathrm{B} \\
& \alpha \mathrm{B}+\delta \alpha \rightarrow \delta \mathrm{B}
\end{aligned}
$$

Concurrently, the leading partial curves around the SFT, detaching.

(4) The trailing partial catches up and reconnects, falling upon the leading partial in the backside $(b)$. This leads to the formation of a perfect $\mathrm{BA} 30^{\circ}$-mixed dislocation on $(b)$.

This is again the observed mechanism up to $h \leq 0.23 h_{0}$, Orowan loops are produced above this height. Inverting the Burgers vector from $\mathrm{AB}$ to $\mathrm{BA}$ simply prevents the dissociation on facet $(a)$ mentioned in step (1).

\subsubsection{Basal interaction mechanisms}

Here we consider the two possible interactions, $\mathrm{AB}$ and $\mathrm{BA}$, and give the details in Appendix B. The summary of the results is:

- $\mathrm{AB}$ reaction (Appendix B.1): the dislocation curves around the base, leaving an Orowan loop.

- BA reaction (Appendix B.2): the SFT is left intact.

\subsubsection{Strength of SFTs to mixed $30^{\circ}$ and edge dislocation passage}

Figure 8 shows the $\left(\tau-h / h_{0}\right)$ curves for $\mathrm{AB}(d)$ and $\mathrm{BA}(d)$ edge dislocations traversing through a $4.7-\mathrm{nm}$ SFT. In the inset to Figure 8 we compare the strengths for 70 and $150-\mathrm{nm}$ segments for the $\mathrm{AB}$ orientation. As for screw and $60^{\circ}$ dislocations, there is a clear proportionality between the data for both lengths. This proportionality is well described by the ratio $\sigma^{\prime} / \sigma=0.51$, which is very close to the line length ratio of $L / L^{\prime}=70 / 150=0.47$ (the prime denotes results for $150-\mathrm{nm}$ dislocations). In other words, the interaction mechanisms do not affect the overall strengths, which are governed by line tension effects, and the $\sigma L$ product is conserved for all lengths (except, likely, when $L \sim h_{0}$ ).

\subsubsection{SFT size effects}

As in Section 2.2.5, in addition to the 4.7-nm data, we have calculated the strengths for 2.5 and 3.6-nm SFTs for the AB orientation and the results, expressed in terms of $\theta_{e}$, are plotted as a function of the "encountered" area $a$ in Figure 9. Again a simple power law of the type $\theta_{e}(a)=B_{e} / a^{n_{e}}$ provides the best fit to the shown data, with $B_{e}=133.10$ and $n_{e}=0.90$. The encountered area can readily be calculated as $a=\frac{\sqrt{3}}{4}\left(h_{0}-h\right)^{2}$. In this 


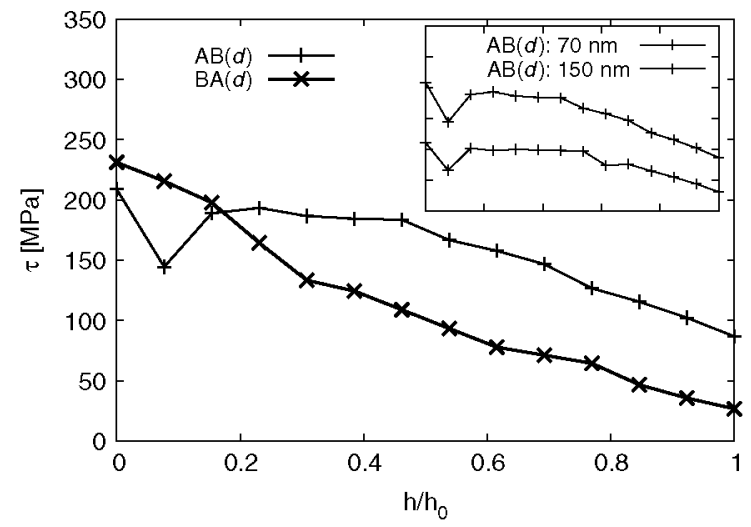

Figure 8. SFT strength as a function of the normalized interaction height, $h / h_{0}$, for $70-\mathrm{nm} \mathrm{AB}$ and BA edge dislocations impinging on a $h_{0}=4.7-\mathrm{nm}$ SFT according to the geometry shown in Figure 1 . The inset shows a comparison between 70 and $150-\mathrm{nm}$ AB dislocations.

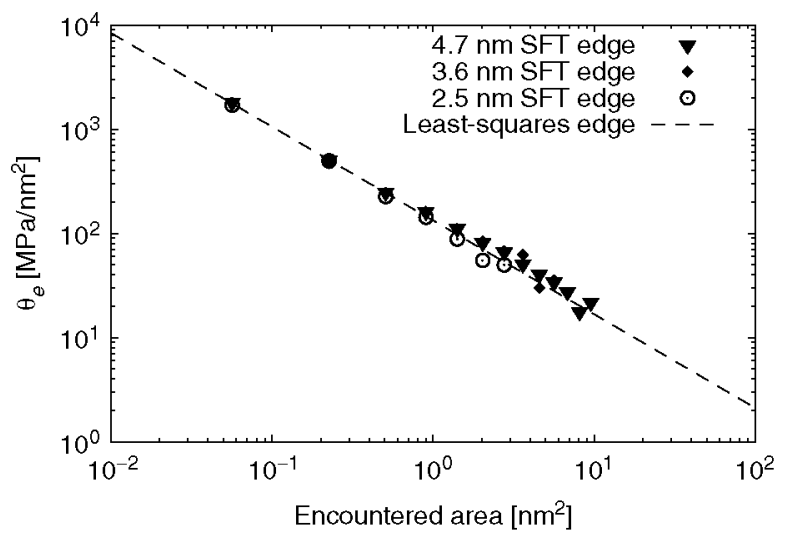

Figure 9. Variation of the stress per unit triangular area, $\theta_{e}$, with the area, $a$, encountered by an edge (or, equivalently, a $30^{\circ}$-mixed) dislocation. The curves show that the SFT strength is independent of the tetrahedron size, and depends solely on the area intersected by the dislocation glide plane. The least-squares fit to the data is $\theta_{e}(\mathrm{a})=133.10 a^{-0.90}$.

fashion, we arrive at the expression for the SFT-size independent strength function for edge and $30^{\circ}$-mixed dislocations:

$$
\begin{gathered}
\theta_{e}(a)=\tau_{e}(a) / a=133.10 a^{-0.90} \\
\tau_{e}(a)=133.10 a^{0.10}
\end{gathered}
$$

\section{Discussion and conclusions}

There are two main conclusions that can be extracted from Part II: (i) SFT strength to dislocation passage depends only on the triangular area resulting from the intersection 


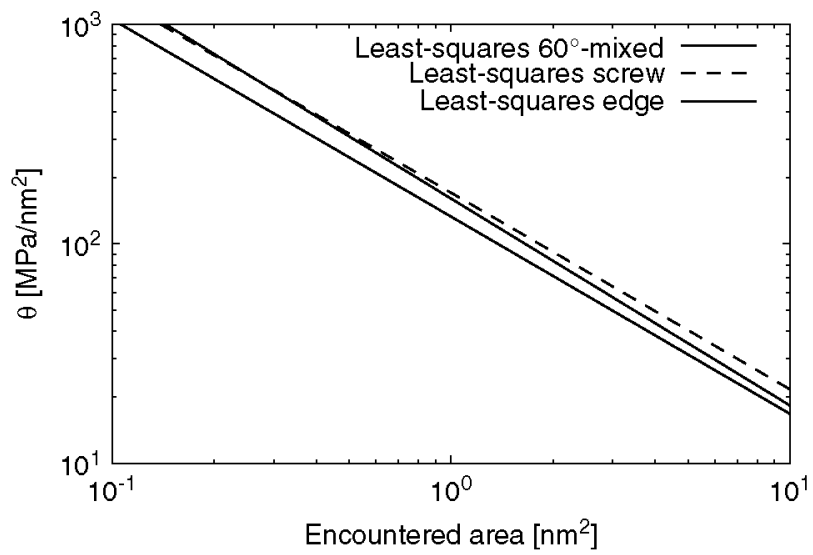

Figure 10. Comparison of the function $\theta(a)$ as a function of dislocation character.

between the SFT and the glide plane; (ii) these strengths can be explained resorting to simple line tension arguments. Together with Part I, Part II serves to generalize these conclusions to screw, edge, and $60^{\circ}$ and $30^{\circ}$-mixed dislocations. We have obtained and fitted simple power laws to compute SFT strengths as a function of the encountered area (for screw and $60^{\circ}$ dislocations, face-on interactions only). Generally, the displayed dependence with the area is weak for all dislocation types (Equations 2 and 4). Furthermore, as Figure 10 shows, the pure edge and screw cases run almost in parallel (very similar power law exponent), whereas the $60^{\circ}$-mixed resembles the edge (or, equivalently, the $30^{\circ}$ ) dislocation behaviour for large areas, and the screw behaviour for small values of $a$.

Our simulations are subject to the same limitations explained in Part I, namely that SFTs cannot physically reduce their size due to the absence of intrinsically-atomistic vacancy-removing mechanisms. Hence, the final structures shown in Figures 3, 4, 7, A1, $\mathrm{A} 2, \mathrm{~A} 3, \mathrm{~B} 1$, and $\mathrm{B} 2$ are not irreversible, and can always find a way back to their original perfect SFT form. Again, we emphasize that this study is one of SFT strength, not stability and/or morphology (although we recognize the importance of this topic and the role that MD simulations have played in it).

Here again, a height-dependent transition from a reaction-governed to an Orowan-loop formation regime is observed in all cases studied. Similar to screw dislocations, this transition does not translate into a discontinuity in the strength curves, further evidence that line tension trumps short-range mechanistic details. Shearing, defined as $f_{i}>f^{*}$, where $f_{i}$ is the force on any dislocation node in contact with a given SFT facet, is never observed. Regarding the dislocation reactions regime - which is observed when the dislocation glide plane is close to, but not at, the SFT base - we conclude that all interactions, independent of dislocation character, can be reduced to two main processes, namely, a Frank-Shockley and a Shockley-Shockley perfect dislocation dissociation. This is owed to the particular geometry of stacking-fault tetrahedra, which lend themselves to only a limited set of possible orientations. Again, regardless of its complexity, the interaction mechanism does not dictate the magnitude of the SFT strength, at least for sufficiently long dislocation segments. The mechanism is important, nonetheless, to understand the morphology of the resulting structures left after dislocation passage, which may affect the subsequent microstructural evolution. 
Basal reactions present their own interesting features. In general, three different mechanisms are observed: (i) the SFT is left intact, (ii) an Orowan loop composed of high-energy stair-rod dislocations is left behind, or (iii) an irreversible structure is produced. These are particularly interesting. For example, the resulting structure seen after the reactions described in Appendix A.4 is akin to a dissociated perfect dislocation loop. The SFT $\rightarrow$ perfect-loop transformation has been assumed in previous DD studies that do not contain partial dislocation resolution and it has been observed experimentally by Robertson et al. in carefully-performed in situ TEM experiments

Equations 2 and 4 can be used in coarser hardening models where the details of the dislocation/SFT interaction are not captured. In this sense, our results show that the quantity $\sigma L$ is conserved for, presumably, $L>h_{0}$. This observation could be used to extend Equations 2 and 4, obtained specifically for 70-nm segments, to arbitrarily-long dislocation lines. For example, for $60^{\circ}$ dislocations, we know the dependence of the $\sigma L$ product with the encountered area:

$$
\sigma_{L} L=160.82 a^{0.06}[\mathrm{MPa}] \cdot 70[\mathrm{~nm}]=11257.4 a^{0.06}
$$

This simple exercise shows that our work can serve as a bridge between high-resolution atomistic simulations and higher-level statistical tools that make use of homogenization techniques where this level of detail is avoided.

Another important conclusion that emanates from Part II is that SFTs are never completely removed by a single dislocation passage. This is in agreement with a number of experimental and simulation works, including some atomistic simulations (although recent TEM experiments also show complete removal ). Despite the relatively large volume of atomistic simulations published in the literature, we have only found one study where quantitative data as a function of the cutting height is given These data were obtained for $\mathrm{BA}(d)$ dislocations, directly comparable with the DD results shown in Figure 8. Comparing the values obtained from both sources meaningfully requires that the different conditions and techniques under which they have been obtained be carefully kept in mind. That way, both data sets can be placed in the right context and plotted directly in the same master curve. In our case, we have just seen that our results suggest that the $\sigma L$ product is conserved for dislocation lengths $L>h_{0}$. In Figure 11, we have converted the MD and DD data to $\sigma L$ and plotted them as a function of $a$. Nevertheless, we want to note the following about the simulation conditions in each case:

- SFT size: 4.2 vs. $4.7 \mathrm{~nm}$.

- Different boundary conditions and dislocation line lengths, $L$.

- Temperature: $10 \mathrm{~K}(\mathrm{MD})$ vs. $100 \mathrm{~K}$.

- Dislocation velocities: $18-340 \mathrm{~m} \mathrm{~s}^{-1}$ (MD) vs. $0.1-10 \mathrm{~m} \mathrm{~s}^{-1}$.

- Existence of core effects and anisotropic elasticity in MD but not in DD.

- Existence of a component of the applied stress resolved on facets $(a)$ and $(c)$ in the MD simulations but not in DD (only on $(d)$ ).

The critical parameter here is $L$, which merits a more elaborate discussion. The MD simulations are done using periodic boundary conditions. This means that the line length available to accumulate tension is the distance between periodic SFTs, which is equal to 


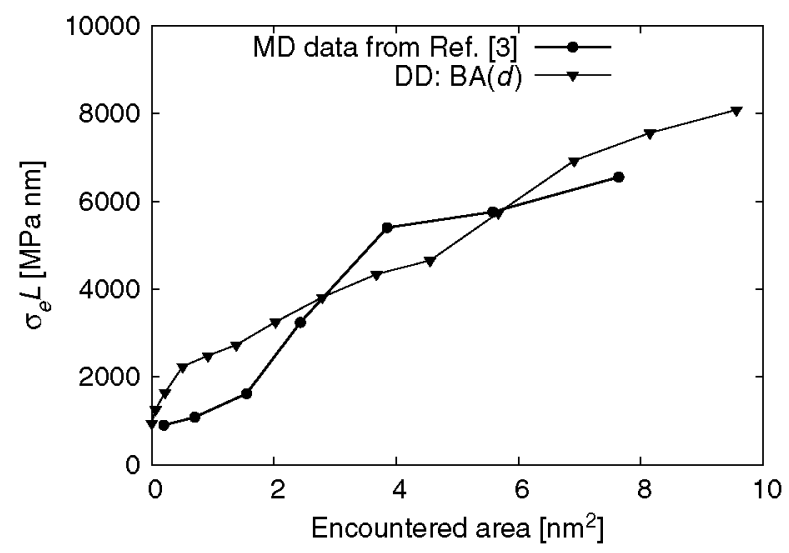

Figure 11. Comparison between $\sigma L$ data obtained from MD and DD (this work) as a function of the traversed area for $\mathrm{BA}(d)$ edge dislocations. The agreement between both data sets is quite remarkable considering the different conditions under which they have been obtained (listed in the text).

the box length along the dislocation line.

In our case, the distance over which the dislocation can curve is $\approx L / 2$ in Figure 2 a (from one end point to the SFT), or, for the BA dislocation data used here, $35 \mathrm{~nm}$. Of course, the manner in which both segments (in MD and DD) accumulate tension is not equivalent due to the existence of fixed end points in DD, but we believe that the agreement shown in Figure 11 is more than reasonable despite the differences listed above. This agreement may be interpreted as a partial validation of the invariance of the $\sigma L$ product, which is also conserved across different techniques.

We want to emphasize that the main objective of our work is not to supplant MD as the main technique to study atomistic-detail mechanisms, but to provide a sufficient degree of atomic-level resolution while retaining the computational expeditiousness required to extract statistical coarsening laws. Large-scale (discrete or stochastic) DD simulations will need to be performed in order to enable a direct comparison with experiments.

\section{References}

E. Martínez, J. Marian, A. Arsenlis et al., J. Mech. Phys. Sol. 56 (2008) p.869.

Y. Matsukawa, Y.N. Osetsky, R.E. Stoller et al., J. Nucl. Mater. 351 (2006) p.285.

Y.N. Osetsky, R.E. Stoller, D. Rodney et al., Mater. Sci. \& Eng. A 400-401 (2005) p.370.

Y.N. Osetsky, Y. Matsukawa, R.E. Stoller et al., Phil. Mag. Lett. 86 (2006a) p.511. 
B.D. Wirth, V.V. Bulatov and T. Díaz de la Rubia, J. Nucl. Mater. 351283 (2000) p.773.

B.D. Wirth, V.V. Bulatov and T. Díaz de la Rubia, J. Eng. Mater. \& Technol.-Trans. ASME 124 (2002) p.329.

H.-J. Lee, J.-H. Shim and B.D. Wirth, Key Engineering Materials 345-346 (2007) p.947.

S.J. Zhou, D.L. Preston, P.S. Lomdahl et al., Science 279 (1998) p.1525.

E. Martínez, J. Marian, A. Arsenlis et al., J. Mech. Phys. Solids, doi:10.1016/j.jmps.2007.06.014.

M. Parrinello and A. Rahman, J. App. Phys. 52 (1981) p.7182.

N.M. Ghoniem, S.H. Tong, J. Huang et al., J. Nucl. Mater. 307-311 (2002) p.843.

T.A. Khraishi, H.M. Zbib, T. Diaz de la Rubia et al., Metall. Mater. Trans. B 33B (2002) p.285.

J.S. Robach, I.M. Robertson, H.J. Lee et al., Acta Mater. 54 (2006) p.1679.

Y.N. Osetsky, D. Rodney and D.J. Bacon, Phil. Mag. 86 (2006) p.2295.

H.J. Lee, J.H. Shim and B.D. Wirth, J. Mater. Res. 22 (2007) p.2758.

M.R. Surowiec, Phys. Stat. Sol. (a) 122 (1990) p.K15.

\section{Appendix A: Basal reactions for the $60^{\circ}$-mixed dislocation}

\section{A.1. Reaction CB face-on}

The interaction is shown in Figure A1. Each frame is explained in detail below:

(1) The leading partial $\delta \mathrm{B}$ reacts with the basal stair-rod $\delta \gamma$ to give a segment with Burger's vector $\frac{1}{6}[301]$ :

$$
\delta \mathbf{B}+\delta \gamma \rightarrow \delta \gamma: \delta \mathbf{B}
$$

This unusual Burgers vector is akin to a Hirth-type dislocation in the sense that both act as a 'hinge', joining two stacking fault regions on the same plane plus another from a secondary plane. As we have mentioned in Part I, the $\delta \gamma: \delta \mathrm{B}$ dislocation is not stable in terms of elastic energy and its stability is contingent upon the applied stress. In its absence, this dislocation is expected to dissociate very rapidly.

(2) The leading partial curves around the SFT base, reacting with the $\delta \alpha$ and $\beta \delta$ stair-rods:

$$
\begin{aligned}
& \delta \mathrm{B}+\delta \alpha \rightarrow \delta \alpha: \delta \mathrm{B} \\
& \delta \mathrm{B}+\beta \delta \rightarrow \beta \delta: \mathrm{B} \delta
\end{aligned}
$$

Again, none of the above is elastically favourable, which contributes to increasing the stress required for dislocation passage through the SFT base. $\delta \alpha: \delta \mathrm{B}$ and $\beta \delta: \mathrm{B} \delta$ are also Hirthtype dislocations and were first observed in Part I.

(3) The trailing partial $\mathrm{C} \delta$ is strongly repelled by the $\delta \gamma: \delta \mathrm{B}$ dislocation. After curving around it, the right-hand arm of $C \delta$ reacts with the existing Hirth segment on the base to form a segment with Burger's vector $\frac{1}{6}[312], \mathrm{CB}: \beta \delta$ in Thompson's notation, that is the sum of a perfect plus a stair-rod dislocation:

$$
\beta \delta: \mathrm{B} \delta+\delta \mathrm{C} \rightarrow \mathrm{CB}: \beta \delta
$$

(4) The left-hand arm of the trailing partial falls upon the existing $\delta \alpha: \delta \mathrm{B}$, giving rise to another uncommon resultant for the Burger's vector: $\frac{1}{3}$ again $\mathrm{CB}: \delta \alpha$ in Thompson's notation.

$$
\delta \alpha: \delta \mathrm{B}+\mathrm{C} \delta \rightarrow \mathrm{CB}: \delta \alpha
$$

The Shockley partials reconnect downstream of the SFT leaving an Orowan loop at the base composed of highly unfavourable dislocation segments. Therefore, it is reasonable to expect that these segments will quickly dissociate as soon as stress is no longer applied. However, these dissociations are not part of our partial dislocation reaction catalogue due to their rarity, and they 

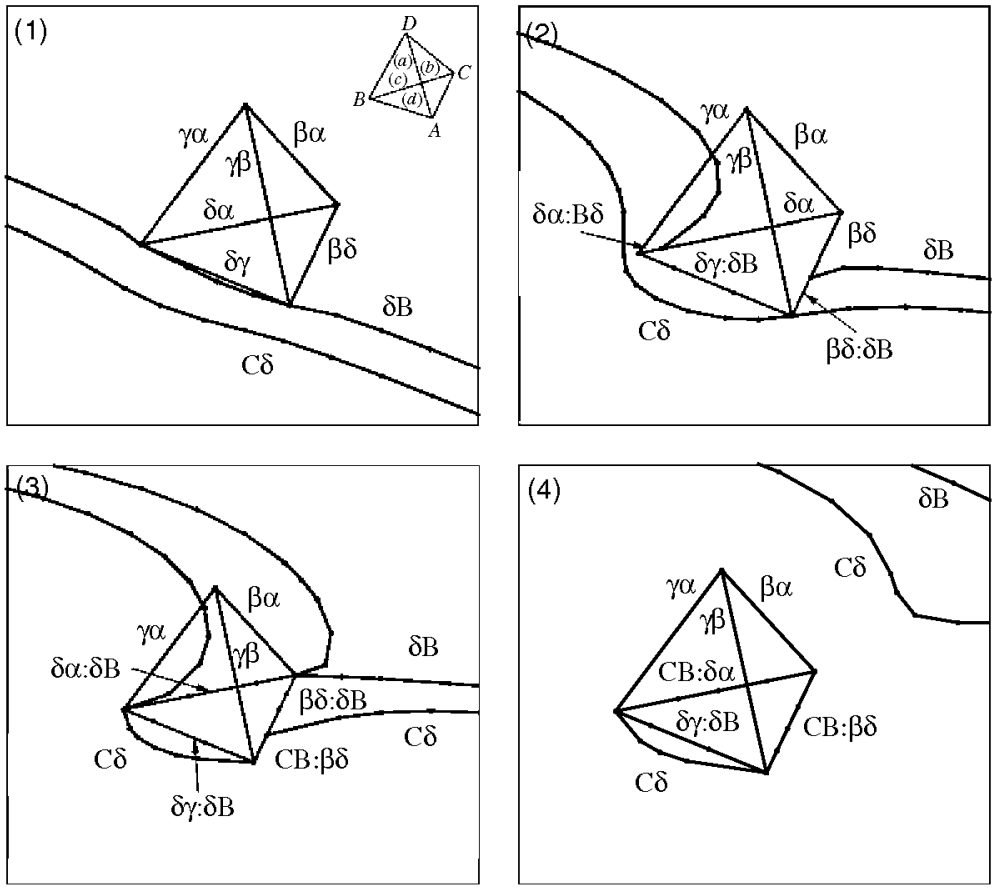

Figure A1. Face-on interaction of a CB $60^{\circ}$ mixed dislocation with the base of a $4.7-\mathrm{nm}$ SFT.

already dissociate during the course of the simulations, when stress is applied. In any case, this is not expected to affect the results in any significant way.

\section{A.2. Reaction $B C$ face-on}

Figure A2 shows the mechanism for this case, itemized below:

(1) In this case, the leading partial is attracted towards the SFT base by the stair-rod $\delta \gamma$, with which it reacts to form a B $\gamma$ Shockley:

$$
\mathrm{B} \delta+\delta \gamma \rightarrow \mathrm{B} \gamma
$$

This partial glides up on the $(c)$ facet of the SFT removing a portion of the stacking fault on it.

(2) The left-hand arm of the leading partial reacts with the $\delta \alpha$ basal stair-rod resulting in a new Shockley, $\mathrm{B} \alpha$, which glides up on the ( $a$ ) facet:

$$
\mathrm{B} \delta+\delta \alpha \rightarrow \mathrm{B} \alpha
$$

The trailing partial keeps gliding, removing the original base as it moves.

(3) The trailing partial reacts then with the $\beta \delta$ stair-rod, giving rise to a Shockley partial $\beta C$ that moves up on the $(b)$ facet:

$$
\delta \mathrm{C}+\beta \delta \rightarrow \beta \mathrm{C}
$$

The Hirth segment $\mathrm{A} \delta: \delta \alpha$ is given by the triple reaction:

$$
\delta \mathrm{B}+\delta \mathrm{C}+\delta \alpha \rightarrow \mathrm{A} \delta: \delta \alpha
$$



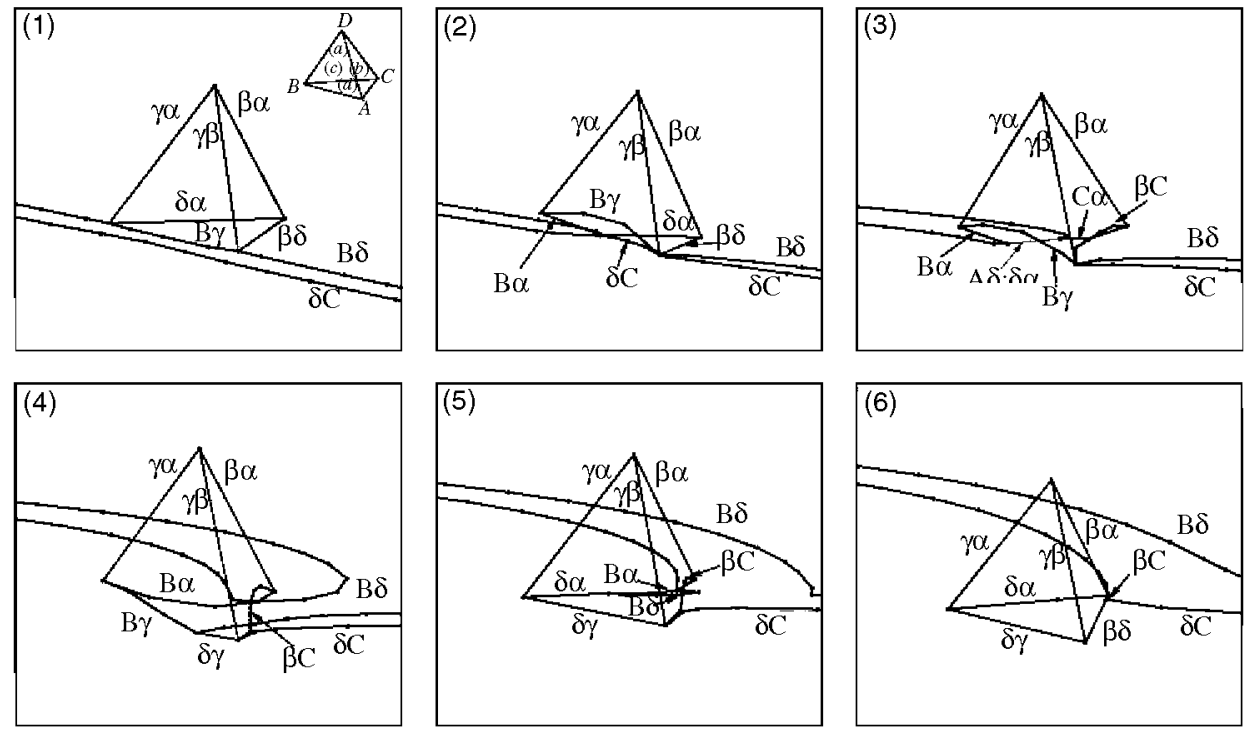

Figure A2. Face-on interaction of a $\mathrm{BC} 60^{\circ}$ mixed dislocation on the base of a 4.7-nm SFT.

(4) Due to the line curvature, the leading partial reverses its sign and reacts with the Shockley B $\gamma$ on facet $(c)$ :

$$
\mathrm{B} \gamma+\delta \mathrm{B} \rightarrow \delta \gamma
$$

Meanwhile, the trailing partial continues to curve around the defect.

(5) The two arms of the leading partial reconnect downstream of the SFT, leaving a segment behind that reacts with $\mathrm{B} \alpha$ on $(a)$ to reform the original stair-rod $\delta \alpha$ :

$$
\delta \mathrm{B}+\mathrm{B} \alpha \rightarrow \delta \alpha
$$

(6) The trailing partial (with its line tangent inverted) and the Shockley gliding on plane $(b), \beta \mathrm{C}$, react, reforming the stair-rod $\beta \delta$ :

$$
\beta \mathrm{C}+\mathrm{C} \delta \rightarrow \beta \delta
$$

Subsequently, both arms of $\delta \mathrm{C}$ meet at the trailing edge of the tetrahedron, leading to full detachment and leaving a reconstructed SFT behind.

\section{A.3. Reaction CB edge-on}

In this case the interaction is quite straightforward, as Figure A3 shows:

(1) The leading partial comes into contact with the base and reacts with the stair-rod on the right-hand side, resulting in a new Shockley partial with Burger's vector $\alpha \mathrm{B}$ :

$$
\delta \mathrm{B}+\alpha \delta \rightarrow \alpha \mathrm{B}
$$

This partial moves up on facet (a), removing the stacking fault in its wake.

(2) The trailing partial hugs the base, sweeping it through the opening left on the right-hand side by the Shockley partial $\mathrm{B} \alpha$. On the left, the portion of $\mathrm{C} \delta$ that is sweeping the base 

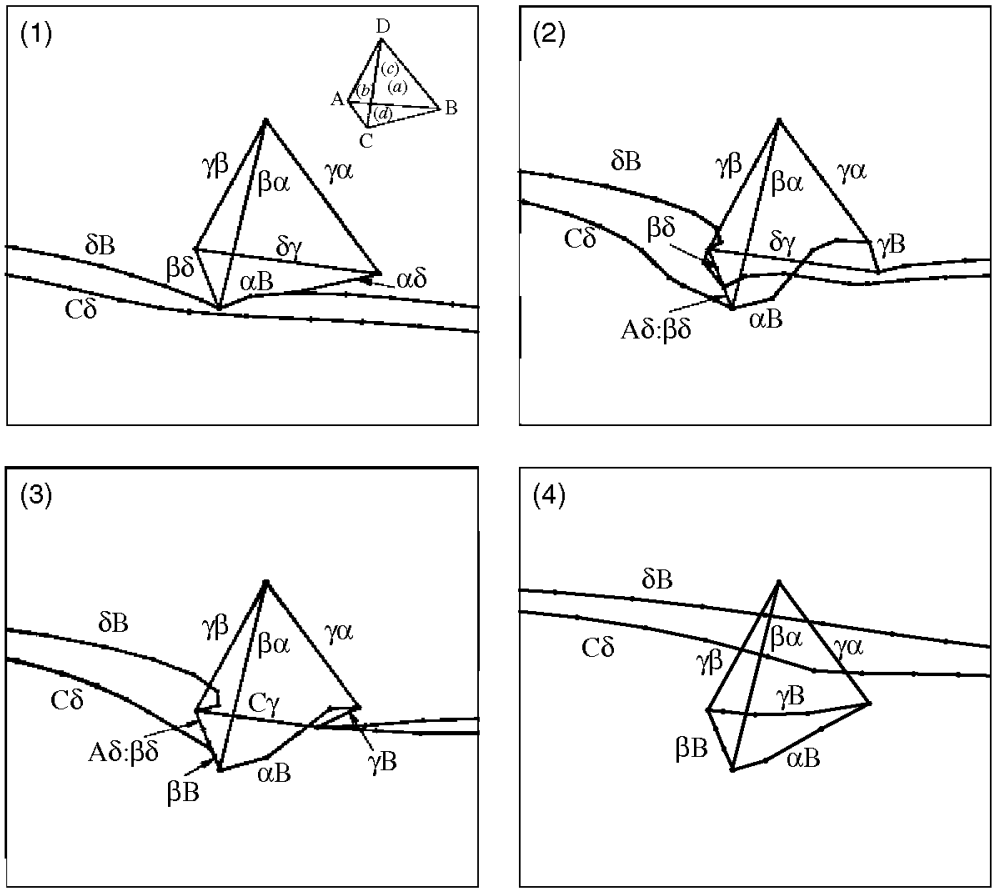

Figure A3. Edge-on interaction of a CB $60^{\circ}$ mixed dislocation on the base of a 4.7-nm SFT.

comes into contact (with reversed sign due to curvature) with the leading partial and with the basal stair-rod $\beta \delta$ :

$$
\delta \mathrm{C}+\delta \mathrm{B}+\beta \delta \rightarrow \delta \mathrm{A}: \beta \delta
$$

which is a Hirth-type dislocation with Burgers vector $\frac{1}{6}[130]$. Meanwhile, $\alpha \mathrm{B}$ reacts with $\gamma \alpha$ giving rise to $\gamma \mathrm{B}$ :

$$
\alpha \mathrm{B}+\gamma \alpha \rightarrow \gamma \mathrm{B}
$$

(3) The portion of the trailing partial $\mathrm{C} \delta$ that swept the base falls upon the backside stair-rod $\delta \gamma$ giving rise to a Frank segment $\mathrm{C} \gamma$ :

$$
\mathrm{C} \delta+\delta \gamma \rightarrow \mathrm{C} \gamma
$$

When the trailing partial collapses onto the $\delta \mathrm{A}: \beta \delta$ segment, a $\beta \mathrm{B}$ Frank segment arises:

$$
\mathrm{C} \delta+\delta \mathrm{A}: \beta \delta \rightarrow \beta \mathrm{B}
$$

(4) The dislocation (one partial followed by the other) curves back around the SFT reacting with the backside Frank segment, resulting in a $\mathrm{B} \gamma$ Shockley:

$$
\mathrm{BC}+\mathrm{C} \gamma \rightarrow \mathrm{B} \gamma
$$

leaving a tetrahedron with the base removed. 

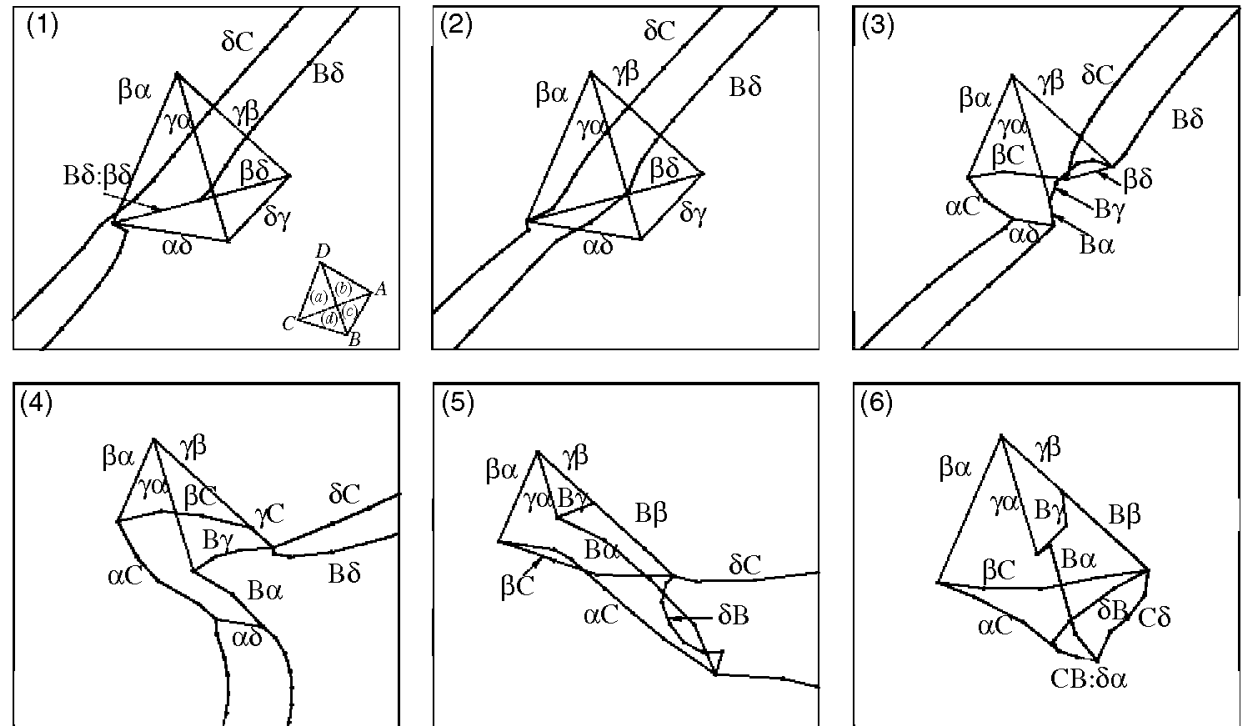

Figure A4. Edge-on interaction of a $(\mathrm{B} \delta, \delta \mathrm{C}) 60^{\circ}$ mixed dislocation and the base of a $4.7-\mathrm{nm}$ SFT.

\section{A.4. Reaction $B C$ edge-on}

Figure A4 shows a sequence of snapshots of the interaction between a $\mathrm{BC} 60^{\circ}$ mixed dislocation and an SFT. The mechanism, itemized below with direct correspondence with the images in Figure A4, is more complicated in this case:

(1) The leading partial reacts with the stair-rod $\delta \alpha$, giving rise to a high-energy Hirth-type segment with Burgers vector $\frac{1}{6}$

$$
\mathrm{B} \delta+\beta \delta \rightarrow \mathrm{B} \delta: \beta \delta
$$

(2) This Hirth segment connects two stacking fault regions on the glide plane plus another one on the secondary $(b)$ plane. What happens next is that this segment dissociates into the original $\beta \delta$ stair-rod and $\delta \mathrm{B}$ partial inside the base. This way the basal stacking fault is removed by this Shockley partial gliding inside the base. The re-formed stair-rod has the same Burgers vector but it now connects obtuse stacking fault planes, rather than acute ones.

(3) When the trailing partial comes into contact with the SFT it reacts with the two corresponding stair-rods, giving rise to new Shockley partials that glide up their respective facets $((a)$ and $(b))$ :

$$
\delta \mathrm{C}+\left\{\begin{array}{l}
\beta \delta \rightarrow \beta \mathrm{C} \\
\alpha \delta \rightarrow \alpha \mathrm{C}
\end{array}\right.
$$

When the leading partial that was gliding inside the base reaches the SFT backside, it reacts with the $\delta \gamma$ stair-rod:

$$
\mathrm{B} \delta+\delta \gamma \rightarrow \mathrm{B} \gamma
$$

This partial glides up on plane $(c)$ and reacts with the stair-rod $\gamma \alpha$ :

$$
\mathrm{B} \gamma+\gamma \alpha \rightarrow \mathrm{B} \alpha
$$


(4) $\beta \mathrm{C}$ reaches the $\gamma_{\beta}$ stair-rod and reacts with it resulting in a $\gamma \mathrm{C}$ Frank segment:

$$
\beta \mathrm{C}+\gamma \beta \rightarrow \gamma \mathrm{C}
$$

On the other side, the small remaining segment of $\delta \alpha$ is literally transported downstream of the SFT.

(5) The $\gamma \mathrm{C}$ Frank dissociates into its constituents $\gamma \beta$ and $\beta \mathrm{C}$. Then, $\gamma \beta$ associates itself with the Shockley partial on (c) B $\gamma$ :

$$
\mathrm{B} \gamma+\gamma \beta \rightarrow \mathrm{B} \beta
$$

Also, this $\mathrm{B} \gamma$ partial continues to react with the $\gamma \alpha$ stair-rod dislocation in the opposite side of facet $(c)$, resulting in a longer $\mathrm{B} \alpha$ segment.

(6) The original dislocation detaches from the SFT leaving a defect which can be analysed in the following terms: (i) the Frank dislocation $\mathrm{B} \beta$ and the Shockley partial $\beta \mathrm{C}$ represent a dissociated perfect $\mathrm{BC}$ segment, which is a Lomer-type dislocation and would be sessile if formed; (ii) the original segments $\delta \mathrm{C}$ and $\mathrm{B} \delta$ lying close to what used to be the $\mathrm{BA}$ edge of the SFT represent a glissile perfect $C B$ dislocation on the original glide plane; (iii) the segments $\mathrm{B} \alpha$ and $\alpha \mathrm{C}$ located on the edges of facet $(a)$ also are equivalent to a dissociated perfect $\mathrm{BC}$ segment. In other words, the structure shown in Figure A4(6) represents a dissociated perfect loop with partial mobility.

\section{Appendix B: Basal reactions for the edge dislocation}

\section{B.1. Reaction of an $A B$ edge dislocation and an $S F T$}

The interaction process is depicted in Figure B1. The different frames are explained below:

(1) The leading partial $\delta \mathrm{B}$ comes into contact with vertex $\mathrm{B}$ of the SFT.

(2) The leading partial reacts with the basal stair-rod $\delta \alpha$, giving rise to a segment with Burgers vector $\frac{1}{6}[3 \overline{10}]$, of the same kind as that reported in Appendix A for $60^{\circ}$-mixed dislocations and in the literature

$$
\delta \mathbf{B}+\delta \alpha \rightarrow \delta \alpha: \delta \mathbf{B}
$$

(3) Likewise, the leading partial reacts with the other basal stair-rods:

$$
\begin{aligned}
& \delta \mathrm{B}+\delta \gamma \rightarrow \delta \gamma: \delta \mathrm{B} \\
& \mathrm{B} \delta+\beta \delta \rightarrow \beta \delta: \mathrm{B} \delta
\end{aligned}
$$

When the trailing partial catches up, it is repelled by the $\delta \alpha: \delta \mathbf{B}$ dislocation but attracted by $\delta \gamma: \delta \mathrm{B}$, with which it reacts giving rise to a high-energy stair-rod dislocation $\mathrm{AB}: \delta \mathrm{B}$ :

$$
\delta \gamma: \delta \mathrm{B}+\mathrm{A} \delta \rightarrow \mathrm{AB}: \delta \gamma
$$

(4) A similar reaction takes place on the CA edge of the tetrahedron, whereby a $\mathrm{AB}: \beta \delta$ is formed. After this, the dislocation detaches leaving an Orowan loop-type structure behind.

\section{B.2 Reaction of a $B A$ edge dislocation and an SFT}

The interaction process is given in Figure B2. The different frames are explained below:

(1) The leading Shockley $\mathrm{B} \delta$ reacts with the basal stair-rods of edges $\mathrm{CB}$ and $\mathrm{BA}, \delta \alpha$ and $\delta \gamma$ respectively:

$$
\mathrm{B} \delta+\left\{\begin{array}{l}
\delta \alpha \rightarrow \mathrm{B} \alpha \\
\delta \gamma \rightarrow \mathrm{B} \gamma
\end{array}\right.
$$





Figure B1. Interaction of an AB edge dislocation with the base of a 4.7-nm SFT.
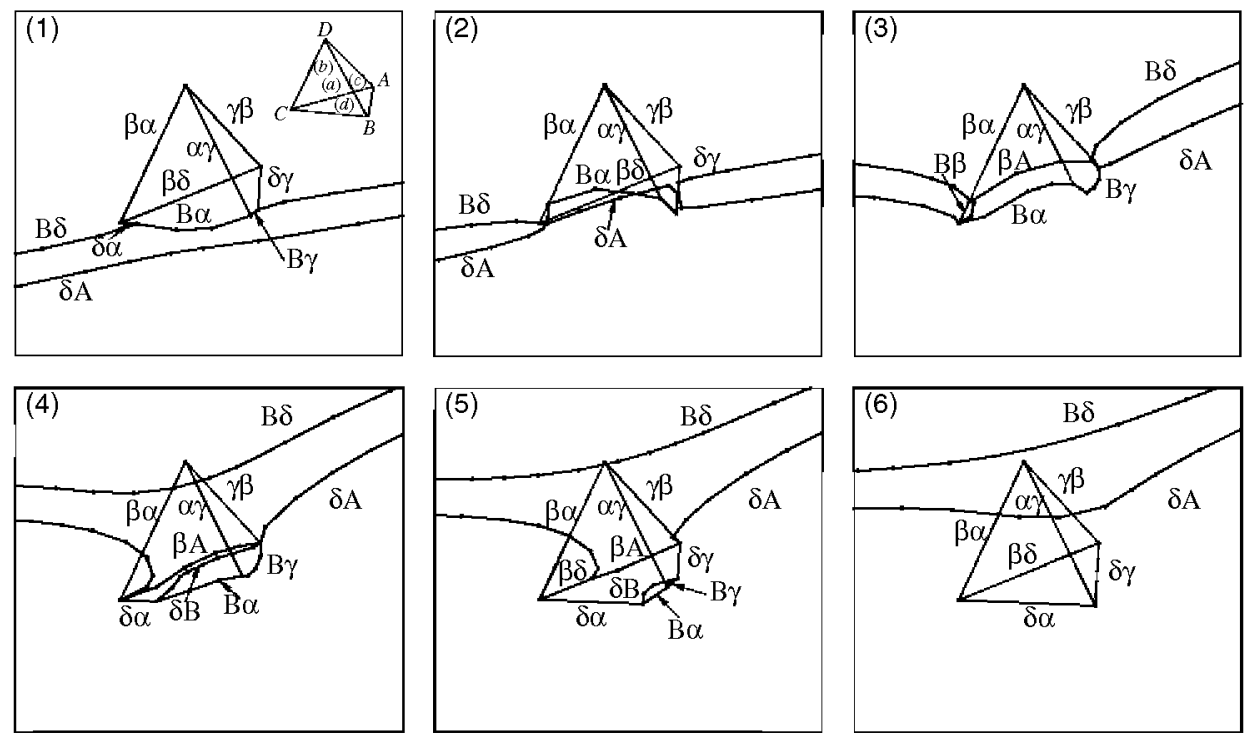

Figure B2. Interaction of an BA edge dislocation with the base of a 4.7-nm SFT. 
(2) These newly-created Shockleys glide up on facets $(a)$ and $(c)$, respectively, eliminating the stacking fault as they proceed. After this, the trailing partial $\delta \mathrm{A}$ is free to sweep the base unimpeded.

(3) When the trailing partial reaches the back edge it reacts with $\beta \delta$ :

$$
\delta \mathrm{A}+\beta \delta \rightarrow \beta \mathrm{A}
$$

which proceeds to glide up on facet $(b)$.

(4) Once the leading partial has reconnected and detached, the $\delta \mathrm{B}$ segment left behind glides backwards towards the original vertex $\mathrm{B}$.

(5) $\delta \mathrm{B}$ forms Lomer-Cottrell junctions with the Shockleys created in step (1):

$$
\delta \mathrm{B}+\left\{\begin{array}{l}
\mathrm{B} \gamma \rightarrow \delta \gamma \\
\mathrm{B} \alpha \rightarrow \delta \alpha
\end{array}\right.
$$

reconstructing the original tetrahedron. At the same time, the curved trailing partial segment A $\delta$ reacts with the Shockley left in the backside:

$$
\mathrm{A} \delta+\beta \mathrm{A} \rightarrow \beta \delta
$$

completing the reconstruction.

(6) The trailing partial reconnects with itself leaving the a fully reconstructed tetrahedron. 